\title{
Ein Interview zur Erfassung sozialer Ängste unter Einbeziehung von Körpersymptomen
}

\author{
Samia Chaker $^{\mathrm{a}}$ Elisa Haustein ${ }^{\mathrm{a}} \quad$ Jürgen Hoyer ${ }^{\mathrm{a}} \quad$ Jonathan R.T. Davidson $^{\mathrm{b}}$ \\ a Institut für Klinische Psychologie und Psychotherapie, Technische Universität Dresden, Deutschland \\ ${ }^{\mathrm{b}}$ Duke University Medical Center, Anxiety and Traumatic Stress Program, Durham, NC, USA
}

\section{Einleitung}

Die Brief Social Phobia Scale - German version (BSPS-G) ist ein Kurzinterview zum Screening sozialer Ängste. Dabei werden Schwere oder Verlauf einer bereits diagnostizierten sozialen Angststörung erfasst. Im Folgenden wird der Hintergrund dargelegt und eine Kurzbeschreibung des englischsprachigen Originals (BSPS) gegeben:

Die soziale Phobie ist gekennzeichnet durch ausgeprägte und anhaltende Angst, sich in sozialen oder Leistungssituationen peinlich $\mathrm{zu}$ verhalten, gedemütigt $\mathrm{zu}$ werden oder bestimmte Körpersymptome zu zeigen (Diagnostisches und Statistisches Manual psychischer Störungen, DSM-IV-TR [Saß et al., 2003]). Die Prävalenzraten liegen zwischen 6,7\% [Fehm et al., 2005] und 12,1\% [Kessler et al., 2005] und bei bis zu 25\% für subklinische Ängste, die in Schwere und Beeinträchtigung häufig dem Vollbild ähnlich sind [Beesdo et al., 2007; Knappe et al., 2009]. Zur diagnostischen Erfassung der sozialen Phobie liegen zahlreiche Selbsteinschätzungsverfahren vor, aber kaum Fremdbeurteilungsverfahren [Mitte et al., 2007]. Als Interviewverfahren hat sich die Liebowitz Skala (Liebowitz Soziale Angst Skala, LSAS) [Stangier und Heidenreich, 2005] etabliert. Die BSPS hat demgegenüber die Vorteile, dass sie viel ökonomischer ist und explizit typische Körpersymptome erfasst, die soziale Ängste sowohl begleiten als auch auslösen können. Die Validierung der englischsprachigen Originalversion der BSPS zeigte sehr gute Kennwerte für die Test-Retest-Reliabilität $\left(r_{t t}=0,91\right)$ und die interne Konsistenz (Cronbachs $\alpha=0,82$ ) sowie gute konvergente und diskriminante Validität und Änderungssensitivität. Als CutOff-Wert wurde ein Summenwert von 20 Punkten bestimmt [Davidson et al., 1997].

\section{Aufbau und Durchführung der BSPS-G}

Die BSPS-G besteht aus den Subskalen «Angst» und «Vermeidung» mit je 7 Items und der Subskala «Körpersymptome» mit 4 Items (gesamt 18 Items). Die Beurteilung erfolgt auf einer fünfstufigen, verankerten Likert-Skala von $0=$ nie bzw. keine bis 4 = immer bzw. sehr stark für die letzten 7 Tage. Zum Beispiel lautet die Verankerung der Subskalen Angst und Körpersymptome für die mittlere Kategorie «häufig» und/oder «etwas Besorgnis erregend», für die Subskala Vermeidung «34-66\%». Zur Auswertung wird ein Gesamtsummenscore gebildet, der einen Bereich von 0-72 Punkten umfasst. Eine einzelne Betrachtung der Subskalen ist ebenfalls möglich.

Die BSPS-G wird als Interview durchgeführt. Der Interviewer sollte Fachkenntnisse zu Symptomen und Diagnostik der sozialen Angststörung besitzen. Er wählt anhand der vorgegebenen Verankerungen die am besten zu den Antworten des Klienten passende Kategorie aus. Hat ein Klient eine Situation in den letzten 7 Tagen nicht erlebt, soll die Einschätzung so erfolgen, als wenn er die Situation erlebt hätte. Die Durchführungsdauer des Interviews beträgt zwischen 2 und 5 min. Die Auswertung durch Aufsummieren dauert 1-2 min.

\section{Übertragung ins Deutsche und erste psychometrische Gütekriterienerhebung}

Entsprechend den Empfehlungen von Beaton und Kollegen [2000] wurde das englische Original von SC und EH unabhängig ins Deutsche übersetzt, beide Versionen wurden von JH und einem weiteren Kollegen überprüft. Ein Muttersprachler nahm eine Rückübersetzung ins Englische vor, die JRTD mit dem Original verglich. Die Inspektion der Item-Skalen-Korrelation ergab keine Hinweise auf systematische Fehler durch Itemformulierungen, weshalb zur internationalen Vergleichbarkeit der deutschen Übersetzung mit der Originalversion alle Items beibehalten wurden. Beurteilerübereinstimmung, interne Konsistenz, Retest-Reliabilität, konvergente und diskriminante Validität sowie Änderungssensitivität wurden an

Dipl.-Psych. Samia Chaker

Technische Universität Dresden

Institut für Klinische Psychologie und Psychotherapie

Hohe Straße 53, 01187 Dresden, Deutschland

Tel. +49 351463 36-963, Fax -984

chaker@psychologie.tu-dresden.de 
einer Stichprobe von Sozialphobiepatienten mit der Hauptbeschwerde «Errötungsangst» $(\mathrm{n}=70)$, die an einer randomisierten kontrollierten Therapiestudie teilnahmen, bestimmt. Das Studienprotokoll wurde von der Ethikkommission der Technischen Universität Dresden genehmigt. Alle Teilnehmer gaben ihre schriftliche Einwilligung. Die Teilnehmer waren im Mittel 37,8 Jahre $(\mathrm{SD}=11,2)$ alt, 56 Teilnehmer $(80 \%)$ waren weiblich, 28 (40\%) hatten einen Realschulabschluss und 42 (60\%) Abitur. Der Mittelwert der BSPS-G lag zu Therapiebeginn bei 35,43 (SD = 7,35; Bereich: 21-53).

Zur Erfassung der Beurteilerübereinstimmung wurde eine Teilstichprobe von $n=13$ Studienteilnehmern von zwei Interviewern befragt. Zur Berechnung wurde der Intraklassen-Korrelationskoeffizient (ICC) herangezogen, der mit ICC $=1,00(\mathrm{p}<0,01)$ eine vollständige Übereinstimmung ergab.

Die Ermittlung der internen Konsistenz erfolgte an der Gesamtstichprobe $(\mathrm{n}=70)$ zu Therapiebeginn. Für die Gesamtskala (18 Items) ergab sich ein guter Wert der internen Konsistenz von Cronbachs $\alpha=0,83$, fü die Unterskala Angst (7 Items) von $\alpha=0,75$, für die Unterskala Vermeidung (7 Items) von $\alpha=0,74$ und für die Unterskala Körpersymptome (4 Items) von $\alpha=0,59$. Die letzte Skala erfasst 4 eher heterogene Körpersymptome, sodass die Annahme einer homogenen Niveauskala nicht unbedingt zugrunde gelegt werden kann.

Die Wartekontrollgruppe der Studie $(n=28)$ wird als Teilstichprobe zur Erfassung der Retest-Reliabilität herangezogen. Die Wartezeit betrug im Durchschnitt 4 Wochen, in denen keine therapeutischen Kontakte stattfanden. Die Retest-Reliabilität betrug $r_{t t}=0,78$ für die Gesamtskala, $r_{\mathrm{tt}}=0,71$ für die Unterskala Angst, $\mathrm{r}_{\mathrm{tt}}=0,79$ für die Unterskala Vermeidung und $r_{\mathrm{tt}}=$ 0,69 für die Unterskala Körpersymptome.

Konvergente und diskriminante Validität wurde für die Gesamtstichprobe $(\mathrm{n}=70)$ zu Beginn der Behandlung mittels Korrelation zu gut etablierten Selbstbeschreibungsinstrumenten erfasst. Die Korrelation zu der LSAS [Stangier und Heidenreich, 2005] lag bei $\mathrm{r}=0,72$, zu der Social Interaction Anxiety Scale (SIAS) [Stangier et al., 1999] bei $r=0,70, \mathrm{zu}$ der Social Phobia Scale (SPS) [Stangier et al., 1999] bei $r=0,57$ und zu dem Social Phobia Inventory (SPIN) [Sosic et al., 2007] bei $r=0,70$. Alle Korrelationen sind signifikant und liegen im mittleren bis hohen Bereich. Die diskriminante Validität wurde anhand der Korrelation zu Depressivität (Beck Depressionsinventar II, BDI-II) [Hautzinger et al., 2006] geprüft. Die Korrelation liegt mit $r=0,46(\mathrm{p}<0,001)$ im moderaten Bereich. Werden zur Korrelation nur diejenigen Studienteilnehmer herangezogen, die nicht an einer komorbiden affektiven Störung litten $(\mathrm{n}=39)$, sinkt die Korrelation auf $\mathrm{r}=0,41(\mathrm{p}=0,011)$.

Zur Prüfung der Veränderungssensitivität [Schuck, 2000] wurden die Effektstärken der Therapie- und der Wartekontrollgruppe verglichen. Die Prä-Post-Effektstärke lag in der Therapiegruppe bei 2,05 (Mittelwert BSPS-G = 18,71; SD = 8,89), in der Wartekontrollgruppe bei 0,18. Zusätzlich lag zur 6-Monats-Nachmessung auch das Außenkriterium «Erfüllung der Diagnosekriterien der sozialen Angststörung» - erfasst mittels CIDI/ DIA-X (Composite International Diagnostic Interview) [Wittchen und Pfister, 1997], einem vollstandardisierten, computerisierten Interview zur Erfassung psychischer Störungen - für $\mathrm{n}=54$ Studienteilnehmer vor Eine ROC (receiver operating characteristic)-Analyse erbrachte als
Fläche unter der Kurve einen Wert von 0,795 (95\% CI: 0,690-0,901). Wird der Cut-Off-Wert von 20 beibehalten, liegt die sensitivität bei $73,1 \%$ und die Spezifität bei $63,9 \%$.

\section{Bisherige Erfahrungen mit der BSPS-G}

Dem Interviewer steht mit der BSPS-G ein Messinstrument zur Erfassung sozialer Ängste zur Verfügung, welches einfach erlernbar, anwendbar und auswertbar ist. Für Betroffene ist es gut verständlich und erleichtert das Ansprechen von sozialen Ängsten und den dazugehörigen Befürchtungen. Es liegen erste Hinweise auf gute Interviewerübereinstimmung, interne Konsistenz, Retest-Reliabilität, konvergente Validität sowie Veränderungssensitivität vor. Mit Einschränkungen gilt dies für die diskriminante und prädiktive Validität. Alle genannten Befunde, insbesondere die zur Bestimmung des CutOff-Wertes der deutschen Version, bedürfen jedoch weiterer Prüfung an großen Normstichproben und an breiter gestreuten Patientenstichproben. Die ökonomische Anwendbarkeit der BSPS-G spricht jedoch vorläufig für ihren Einsatz zu Screeningzwecken. Für die Anwendung in der psychotherapeutischen Praxis gilt, dass standardisiertes Abfragen der körperlichen Symptome ein wichtiger Schritt sein kann, um den Umgang mit diesen bei der Fallkonzeption angemessen zu berücksichtigen [Chaker et al., 2010; Chaker und Hoyer, 2007]. Die BSPS-G ist von Jonathan R.T. Davidson unter jonathan.davidson@duke.edu erhältlich.

\section{Dank}

Wir danken Stefan G. Hofmann und Andrew T. Gloster für ihre Hilfe bei der Übersetzung und Rückübersetzung des Fragebogens. Die Studie wird durch Drittmittel der Deutschen Forschungsgemeinschaft DFG unter Projektnummer HO1900/5-1 gefördert.

\section{Disclosure Statement}

Samia Chaker, Elisa Haustein und Jürgen Hoyer erklären, dass keine Interessenskonflikte vorliegen. Jonathan R.T. Davidson wurde von Glaxo SmithKline, Physicians Postgraduate Press, Sanofi-Aventis und Euthymics Bioscience für nicht mit dieser Arbeit in Zusammenhang stehende Aktivitäten unterstützt.

\section{Literatur}

Beaton DE, Bombardier C, Guillemin F, Ferraz MB:

Guidelines for the process of cross-cultural adaptation of self-report measures. Spine 2000;25:31863191.

Beesdo K, Bittner A, Pine D et al.: Incidence of social

anxiety disorder and the consistent risk for secondary depression in the first three decades of life. Arch Gen Psychiatry 2007;64:903-912.
Chaker S, Hoyer J: Erythrophobie: Störungswissen und Verhaltenstherapie. Verhaltenstherapie 2007; 17:183-190.

Chaker S, Hofmann SG, Hoyer J: Can a one-weekend group therapy reduce fear of blushing? Results of an open trial. Anxiety Stress Coping 2010;23: 303-318.
Davidson JRT, Miner CM, De Veaugh-Geiss J et al.: The Brief Social Phobia Scale: a psychometric evaluation. Psychol Med 1997;27:161-166.

Fehm L, Pelissolo A, Furmark T, Wittchen HU: Size and burden of social phobia in Europe. Eur Neuropsychopharmacol 2005;15:453-462.

Hautzinger M, Keller F, Kühner C: BDI-II BeckDepressions Inventar Revision. Frankfurt/M., Harcourt Test Services, 2006 
Kessler RC, Berglund P, Demler O et al.: Lifetime prevalence and age-of-onset distributions of DSM-

IV disorders in the National Comorbidity Survey

Replication. Arch Gen Psychiatry 2005;62:593-602.

Knappe S, Beesdo K, Fehm L, Lieb R, Wittchen HU

Associations of familial risk factors with social fears and social phobia: evidence for the continuum hypothesis in social anxiety disorder? J Neural Transm 2009;116:639-648.

Mitte K, Heidenreich T, Stangier U: Diagnostik bei Sozialen Phobien. Göttingen, Hogrefe, 2007.
Saß H, Wittchen HU, Zaudig M, Houben I: Diagnostisches und Statistisches Manual Psychischer Störungen - Textrevision - DSM-IV-TR. Göttingen, Hogrefe, 2003.

Schuck P: Designs und Kennziffern zur Ermittlung der Änderungssensitivität von Fragebogen in der gesundheitsbezogenen Lebensqualitätsforschung. Z Med Psychol 2000;3:125-130.

Sosic Z, Gieler U, Stangier U: Sreening for social phobia in medical in- and outpatients with the German version of the Social Phobia Inventory (SPIN). J Anxiety Disord 2007;22:849-859.
Stangier U, Heidenreich T: Die Liebowitz Soziale Angst Skala (LSAS); in Collegium Internationale Psychiatriae Scalarum (Hrsg): Internationale Skalen für Psychiatrie. Göttingen, Hogrefe, 2005, pp 299-305.

Stangier U, Heidenreich T, Berardi A, Golbs U, Hoyer J: Die Erfassung sozialer Phobie durch die Social Interaction Anxiety Scale (SIAS) und die Social Phobia Scale (SPS). Zeitschrift Klin Psychol 1999;28:28-36.

Wittchen HU, Pfister H: Diagnostisches Expertensystem für Psychische Störungen (DIA-X-Interview). Frankfurt/M, Pearson Assessment, 1997. 